\title{
SEISMIC INPUT IN THE STRUCTURAL DESIGN: CONSIDERATIONS AND APPLICATION TO THE ITALIAN TERRITORY
}

\author{
P. CLEMENTE, G. BONGIOVANNI, G. BUFFARINI \& F. SAITTA \\ ENEA, Rome, Italy.
}

\begin{abstract}
Some recent seismic events pointed out the necessity to revise our way of seismic analysis of structures. But what do structural engineers need to design anti-seismic structures? The first step is the analysis of the seismic input, defined by means of the basic hazard analysis and of the local seismic response. Once the description of the seismic hazard is known, the choice of the protection degree should be made on the basis of economical evaluations. Furthermore, the uncertainties about the local seismic response could be very important. The second step is the structural modeling and analysis, whose suitability depends on how good is the description of the material behavior, geometrical characteristics of the structure, and its behavior both in elastic and inelastic ranges. This is accounted for by means of the behavior factor, whose definition and use are quite delicate and often not necessary, especially in lowto medium-seismicity areas, as demonstrated by means of a comprehensive numerical analysis on the construction costs of reinforced concrete buildings. In this article, some important issues are discussed and some proposals are given to improve the technical codes and hence the safety of structures against earthquakes. The seismic input to be used in the retrofit of existing buildings is also discussed.
\end{abstract}

Keywords: Anti-seismic design, seismic hazard, structural safety.

\section{INTRODUCTION}

Our knowledge about earthquakes and their effects on the structures has improved very much in the last decades, but recent seismic events demonstrated that the way to an acceptable safety level is still very long. The first step in the structural design is the analysis of actions that can affect the structure. Among these, the seismic actions influence very much the structural choices. They are defined by means of the basic hazard analysis and by the local seismic response. The uncertainties about the local effect evaluations could be more significant than those related to the basic analysis. Some recent seismic events questioned several assumptions of the present design codes. For example, during the 2009 L'Aquila earthquake, Italy $\left(M_{W}=6.3\right)$ peak ground accelerations ranging from 0.30 to $0.65 \mathrm{~g}$ were recorded at the stations very close to the city, i.e. at zero Joyner-Boore distance from the fault $[1,2]$. These exceeded those assumed in the design of ordinary buildings, which refer to the return period of 475 years, and were comparable with those relative to the return period of 2475 years. The corresponding response spectra showed the same exceedance with reference to the elastic spectra of the code.

The second step refers to the structural modeling, i.e. to the material and structural behavior, both in elastic and inelastic ranges. These influence the value of the behavior factor assumed in the analysis, which is a measure of the inelastic capacity of the building, i.e. its capacity to dissipate energy. One should expect that, under earthquakes stronger that the design earthquake, most of the structures would collapse or show severe damage, especially if built with reference to old codes or without any code. Actually, and fortunately, this did not happen, demonstrating that our knowledge about the actual behavior of structures under seismic loading is still not satisfactory. 
In this context, it is worth pointing out that the proliferation of codes could be dangerous. In fact, a large number of rules could cause the impoverishment of autonomy and creativity, the difficulty to discern what is really important, and the feeling of being relieved of responsibilities. These concepts, written by Pozzati [3], are still present and agree with the famous motto 'the regulations for design are to regulate those who cannot design'. This motto has several meanings, but the prime meaning is that the art of good designing is much more than just following the prescriptions of the codes.

In this article, some issues about the evaluation of the seismic hazard in the context of the structural design are discussed and some proposals for the future technical codes are provided. Some implications of the suggestions are analyzed with reference to the Eurocode and/ or the Italian technical code but are valid or can be easily extended to almost all the codes in the world.

\section{SEISMIC HAZARD AND LOCAL RESPONSE}

The correct and complete description of the seismic input for structural design at a given site is given by the acceleration components along three orthogonal axes, two horizontal and one vertical, recorded during a suitable number of real events at the site. In practical applications, when using linear analysis, the horizontal and vertical response spectra at the site are used to determine the maximum seismic effects on structures.

In the absence of specific data for the site, one can refer to the horizontal acceleration response spectrum given by the local technical code, which is usually given for a rigid soil with horizontal surface and refers to a conventional damping ratio $\xi=5 \%$. It assumes the shape of Fig. 1. The spectrum amplitudes are defined by means of the horizontal peak ground acceleration $(P G A$, i.e. the value at the period $T=0)$; the maximum amplification $F$ (i.e. the ratio between the maximum amplitude of the spectrum and that at $T=0$ ); and the value $T_{C A}$ (we use this symbol for the period $T_{C}$ on rigid soil) of the period, i.e. the upper limit of the range in which the spectral acceleration is constant. The other parameters can be derived from these.

There are fundamentally two different approaches to determine the spectral amplitudes, the probabilistic seismic hazard assessment (PSHA) and the deterministic seismic hazard assessment (DSHA). The first is based on the seismic history, on the return period concept, and on the knowledge of existing faults, capable or not, and does not account for high magnitude events, whose occurrence has been assessed in the prehistoric age. In the second one,

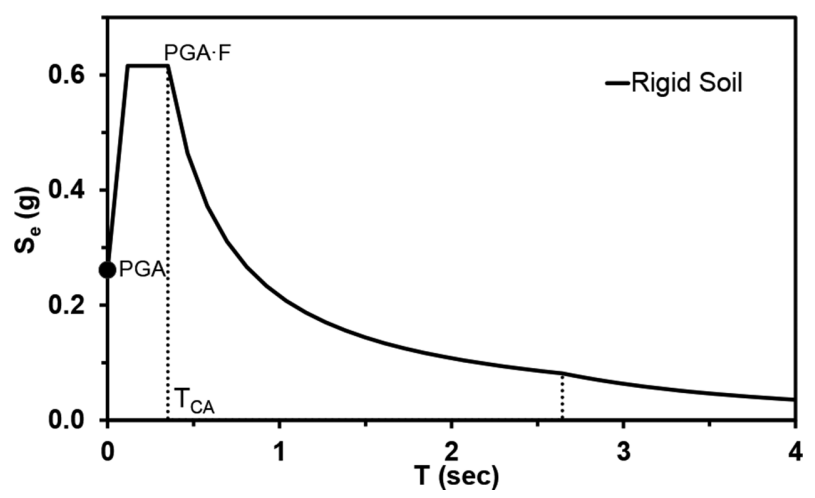

Figure 1: Usual elastic acceleration response spectrum shape. 
individual earthquake scenarios are developed for each relevant seismic source, and also paleoseismic sources are considered, if known; then a ground motion is selected accounting for the maximum credible earthquake $(M C E)$, the distance from the site and its geological characteristics.

According to the PSHA, PGA is given for each site as a function of the probability of exceedance $P_{N C R}$ in 50 years, this being also the minimum service life time usually accepted for structures. $P_{N C R}$ is related to the return period $T_{N C R}$, which can be interpreted as the average time interval between two consecutive events with the same PGA. So PSHA provides a set of acceleration response spectra, each one relative to a return period in the range up to $T_{N C R}=2475$ years, which corresponds to the probability of exceedance $P_{N C R}=2 \%$ and represents a limit related to our knowledge about the seismic history. It is important pointing out that, in order to have reliable data about earthquakes with a certain return period $T_{N C R}$, our observation time should be much longer than $T_{N C R}$ [4]. As a result, because our knowledge about historical earthquakes is limited to the last 2000 years or little more, we should consider return periods consistent with this limit only. The extrapolation to longer periods is done accounting for the uncertainties in order to be on the safer side.

The deterministic approach refers to the maximum credible event $(M C E)$, able to produce the highest level of shaking considered reasonable at the site. The method takes into account both the historical seismicity, i.e. the observed events, and the characteristics of the sources that may affect the site, and determine the design earthquake in terms of magnitude, distance, and focal mechanism. The DSHA does not take into account the recurrence rate of earthquakes, but considers the scenarios that can generate the highest level of shaking at the site, while the PSHA takes into account all the possible shaking scenarios, including those of DSHA [5].

A recent version of the deterministic approach has been proposed, the neo-deterministic seismic hazard analysis (NDSHA). Starting from the allowable information about the lithosphere structure, the distribution and the characteristics of seismic sources and the level of seismic activity in the area, and using numerical models to simulate the wave generation and propagation, the method allows calculation of synthetic seismograms from which one can deduce the earthquake parameters [6]. As the authors stated, the lack of knowledge about the internal structure of the earth does not allow the calculation of components with a frequency higher than $1.0 \mathrm{~Hz}$. The extension to higher frequencies, which are of interest for most of the civil structures, is made taking into account the results of the PSHA. The authors also gave a comparison between the peak ground accelerations of $P S H A$ for $P_{N C R}=2 \%$ in 50 years $(P G A)$ and those of DSHA [Deterministic Peak Ground Acceleration (DGA)] in the Italian territory, showing that the first ones are higher than the second ones at almost all the sites [7].

It should be noted that less frequent events, with a return period longer than 2475 years, could be unknown to us. Furthermore, same faults could not be identified yet, such as the one that generated the earthquake that, on July 16, 2007, struck the town of Chuetsu, Niigata prefecture, in Japan $(M=6.6)$, where the Kashiwazaki-Kariwa nuclear power plant is located, the first in the world with a third-generation reactor and the first to suffer a strong earthquake.

It is important to remind that the seismic waves can be amplified due to site effects. This is well represented by the example of Fig. 2, where the receiver functions at three locations very close to the others, obtained during an aftershock of the 2009 L'Aquila earthquake, are plotted. They are very different, both in terms of ratio amplitudes and frequency content [8].

In the technical codes, this effect is accounted for by multiplying $P G A$, and so the entire spectrum, by the soil coefficient $S$, which decreases when $P G A \cdot F$ gets higher according to a function that depends also on the soil type. Hence, the amplification of motion assumes a very important role especially for lower values of the maximum spectral amplitude and 


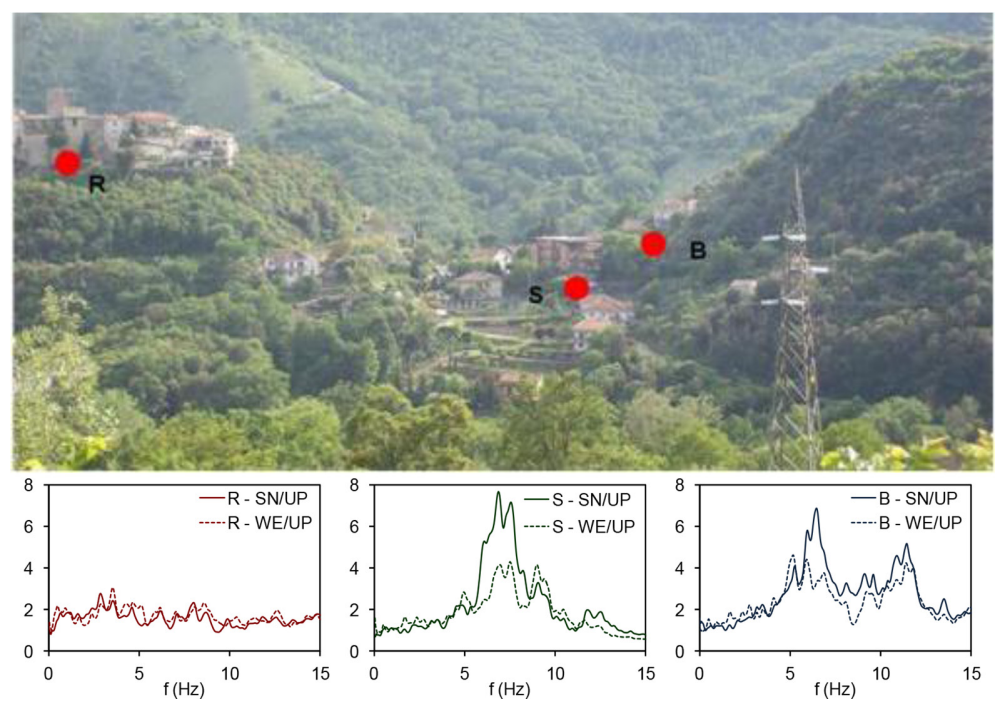

Figure 2: Receiver functions of aftershocks of 2009 L'Aquila earthquake recorded in Belmonte Castello, Italy, at locations R (reference station), S, and B.
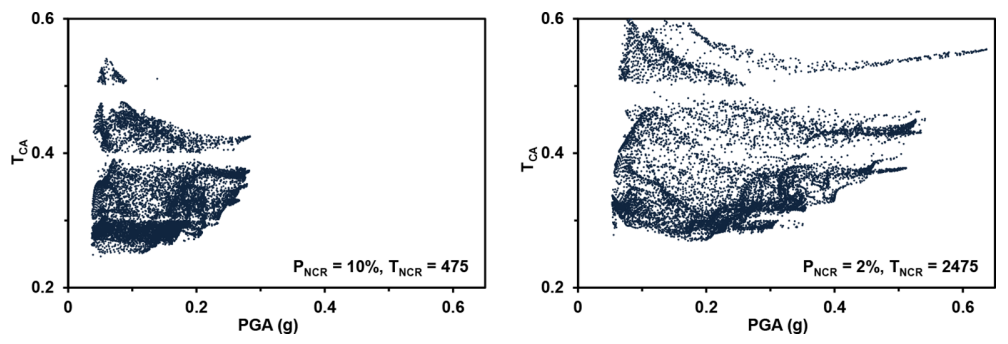

Figure 3: Couples $T_{C A}-P G A$ in the Italian territory, for $P_{N C R}=10 \%$ and $P_{N C R}=2 \%$.

therefore of $P G A$. It is worth reminding that local effects due to slope can also determine an amplification of motion and are also accounted for by means of an amplification coefficient, which ranges up to 1.4 .

The range of maximum amplitude in the acceleration spectrum varies from site to site and depends also on the soil characteristics. The first aspect is usually accounted for by means of the period $T_{C A}$, which separates the range with constant maximum acceleration $\left(T<T_{C A}\right)$ from the range with constant velocity $\left(T>T_{C A}\right)$ in the spectrum relative to rigid soil. The couples $T_{C A}-P G A$ of the Italian territory are plotted in Fig. 3 for the two cases of $P_{N C R}=10 \%$ $\left(T_{N C R}=475\right)$ and $P_{N C R}=2 \%\left(T_{N C R}=2475\right)$, showing an important increment in the second case in comparison to the first. Furthermore, the range of maximum amplification becomes larger for soft soils; hence, $T_{C A}$ is substituted by $T_{C}>T_{C A}$ for non-rigid soils. In Fig. 4, the curves of $T_{C}$ against $T_{C A}$ (continuous lines) and those of the ratio $T_{C} / T_{C A}$ against $T_{C A}$ (dotted lines) are plotted for different soil types, according to the Italian code.

It is apparent that local effects can determine very large variations of the soil motion, as shown in Fig. 5. These variations could be much higher than the uncertainties in the evaluation of the base hazard on rigid soil. 


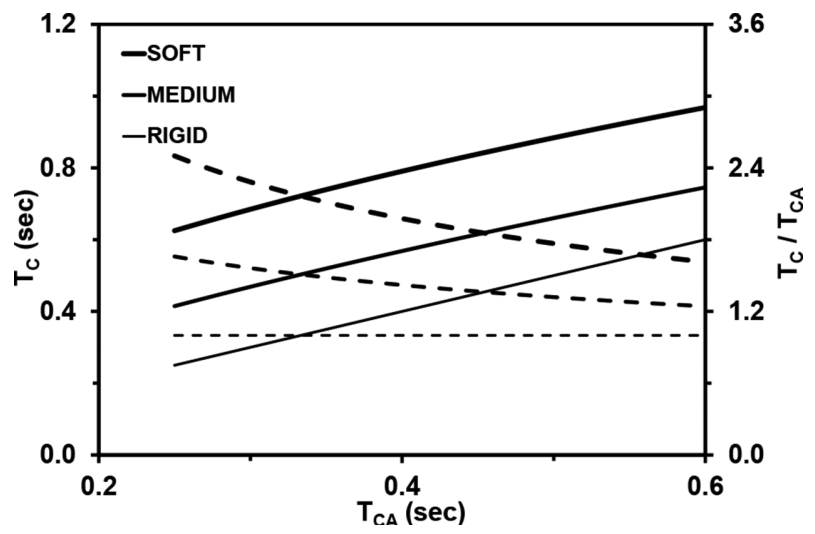

Figure 4: $T_{C}$ (continuous) and $T_{C} T_{C A}$ (dotted) versus $T_{C A}$ for the different soil types.

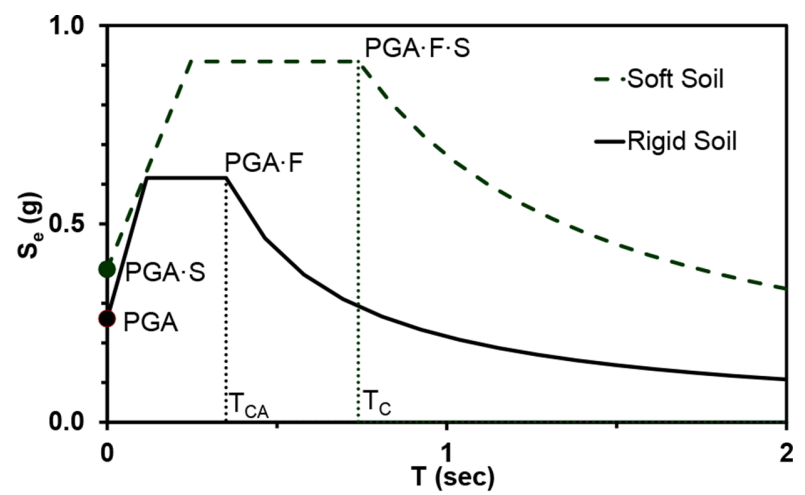

Figure 5: Comparison between the elastic spectra on rigid soil (continuous) and on soft soil (dotted) at the same site.

\section{DESIGN EARTHQUAKE AND BEHAVIOR FACTOR}

According to most of the technical codes, the earthquake intensity to be considered in the structural design, i.e. the return period $T_{N C R}$, is related to the design working life $T_{L}$ and to the importance of the structure. This second aspect is accounted for by means of the importance factor $\gamma_{I}$, which relates to the consequences of a structural failure and is used to amplify the spectral amplitude directly or the design working life $T_{L}$ (as in the Italian code). We think that the design working life of the structure should be an architectural concept only, related to the suitability of the structure in satisfying its intended use, but safety should not depend on it. On the other hand, the design working life of a structure is related to its importance, often because of its high construction cost (consider the case of a long-span bridge); so the safety can be amplified by means of the importance factor $\mathrm{w}_{I}$ as well.

A suitable prevention policy should impose the assumption of the most severe seismic actions for the structural design, independent of $T_{L}$. This translates to considering the minimum value for the probability of exceedance in 50 years, $P_{N C R}=2 \%\left(T_{N C R}=2475\right.$ years $)$, of the reference seismic action for the no-collapse requirement or the $M C E$, which is the event 
of highest intensity expected in the area, determined by the probabilistic and deterministic approaches, respectively.

In practice, due to economic and architectural reasons, a lower seismic intensity is usually accepted. With reference to the PSHA, we can consider in the structural design a value of $P_{N C R}\left(\right.$ or $T_{N C R}$ ) on the basis of the risk level we accept. Analogously, if the deterministic approach is used, a certain percentage of the seismic effects associated with the MCE should be used. The choice requires a detailed knowledge of the behavior of structures under seismic actions and their capability to dissipate the earthquake energy and, therefore, the contribution of engineers is necessary. However, in the end, it is a political issue, because the risk we accept depends on how much we can invest on our safety.

Usually, the probability of exceedance $P_{N C R}=10 \%$ in 50 years is assumed for ordinary buildings, which corresponds to $T_{N C R}=475$ years, as recommended by Eurocode 8 . This should be a conscious choice, which entails a certain risk. Actually, in the Italian territory, the $P G A s$ relative to $P_{N C R}=2 \%$ are between 1.37 and 2.36 times those relative to $P_{N C R}=10 \%$ (Fig. 6), with an average ratio of 1.8. This assumption could be justified for normal structures, in which a certain level of damage could be accepted under strong earthquakes, the goal being the life safeguard. In these cases, a suitable ductility should be assured, and buildings should be designed accounting for a certain capacity of the structure to dissipate energy during earthquakes. This implies that under the design earthquake the structure should not collapse but could suffer damage and behave similarly in the case of events stronger than the design earthquake.

During the 2012 Emilia earthquake, a peak acceleration on surface was recorded at Mirandola. It corresponded to $P G A \approx 0.20 \mathrm{~g}$ at the bedrock. Considering the $P G A s$ expected at Mirandola according to the Italian reference seismic hazard (Fig. 7), the measured value could be associated with an exceedance probability $P_{N C R}<5 \%$ in 50 years, and therefore, it is higher than the design value for ordinary buildings (corresponding to $P_{N C R}=10 \%$ in 50 years). Furthermore, the territories of the Pianura Padana Emiliana have been included in the seismic areas only recently, in 2003. Hence, most of the existing buildings had been designed without accounting for the seismic actions. This is the reason why several industrial buildings were seriously damaged or collapsed. In some cases, they were not statically determinate under horizontal actions or the joints between pillars and beams were not able to transmit even low horizontal forces [9]. The experience of Emilia earthquake taught us that

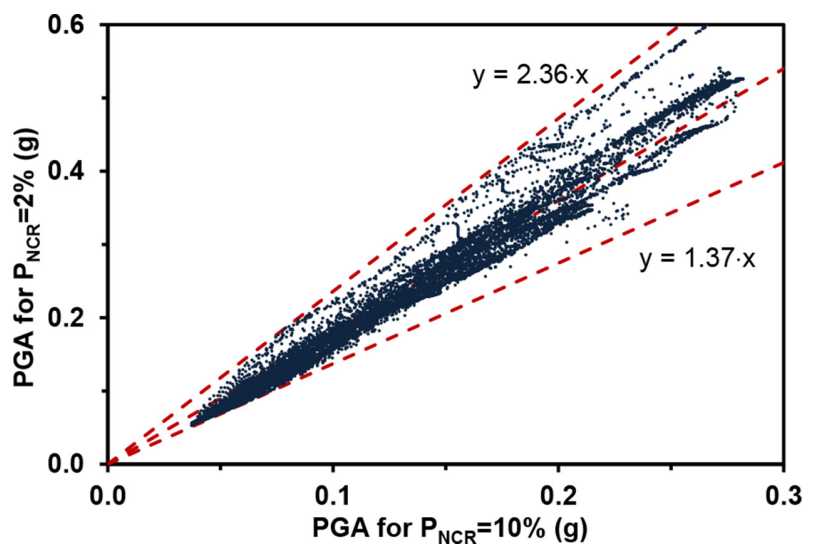

Figure 6: Couples of PGA relative to $P_{N C R}=10 \%$ and $P_{N C R}=2 \%$ in the Italian territory. 


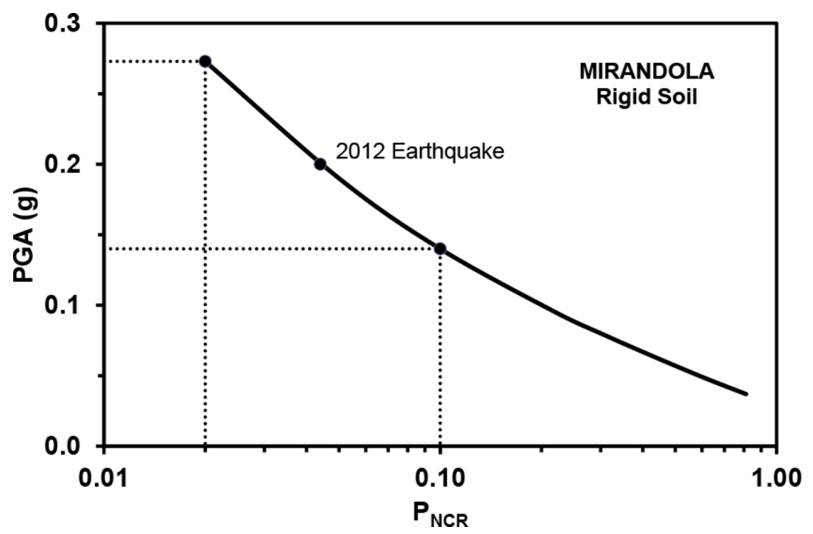

Figure 7: $P G A$ versus $P_{N C R}$ for the Mirandola site according to the Italian seismic hazard.

the highest safety degree should be used for structures of industrial buildings, especially for those of national interest, on which the economy of a wide area depends.

Speaking of ductility, some considerations about the behavior factor are due. In high seismicity areas (HSA), designing structures able to support the effective seismic actions in the elastic range, i.e. without damage, is not suitable both for economic and architectural reasons. Therefore, structures are usually designed by referring to the design spectrum, in which the amplitudes are reduced with reference to the elastic spectrum, by means of the behavior factor $q$. This accounts for the capacity of the structure to dissipate energy during the earthquake and depends on the structural type and on the characteristics of the structural details. In medium- and low-seismicity areas (MSA and LSA, respectively), instead, designing without any reduction of the elastic acceleration values would be possible, also from economical and architectural points of view.

In order to analyze the economic and architectural issues that limit the use of the elastic design, an economic analysis on the construction cost of the carrying structure was performed with reference to a framed reinforced concrete building. It is obviously impossible to obtain general results that can be valid for all the structures. Hence, in order to point out the main aspects of the comparison, the building with a very regular shape in Fig. 8 was analyzed. Different heights (number of stories up to 8) and two different solutions for the number of columns were considered, and therefore, the spaces between them were of two types: type A short spans (Fig. 8, left) and type B long spans (Fig. 8, right).

In all the cases, the same materials have been used for all the structural elements: The concrete has a characteristic compression strength $R_{c k}=30 \mathrm{~N} / \mathrm{mm}^{2}$; the compression design strength at the ultimate limit state is $f_{c d}=14 \mathrm{~N} / \mathrm{mm}^{2}$; the steel of the reinforcing bars has a tensile characteristic strength $f_{y k}=450 \mathrm{~N} / \mathrm{mm}^{2}$; the tensile design strength at the ultimate limit state is $f_{y d}=390 \mathrm{~N} / \mathrm{mm}^{2}$.

All the floor slabs have a thickness equal to $25 \mathrm{~cm}(20+5)$. The buildings have been designed following the same design criteria both for the concrete sections and for the reinforcing steel area, both for the superstructure and the foundation. The structural analysis and the design have been carried out according to the Italian seismic code. The structure is subject to the usual permanent loads (self-weight and other permanent loads) and to the typical variable loads of the residential buildings $\left(2.0 \mathrm{kN} / \mathrm{m}^{2}\right)$. 
The significant sections of all the beams (end-span and half-span sections) and the end sections of all the columns were first designed, on the basis of the internal forces. Then a suitable homogenization was done, on the basis of the following rules:

- The columns at each level have the same concrete cross-section but can have different reinforcement steel areas; obviously, from one level to the other also the concrete crosssections can be different;

- The beams of each floor have the same concrete cross-section but can have different reinforcement steel areas; from one floor to the other also concrete cross-sections can be different;

- The foundation beams have the same concrete cross-section but can have different reinforcement steel areas.

The soil was supposed to be composed by sand with medium geotechnical characteristics. Actually, the soil characteristics could determine an increase in the foundation size but this happens also for static loads only. In Fig. 9 (left), the costs relative to the elastic design $(q=1)$ and the design with a behavior factor $q=4$ are compared for the two column distribution cases with six floors. As one can see the increment of the cost is about $20 \%$ for $S_{e}=0.4 \mathrm{~g}$ and not higher than $40 \%$ for $S_{e}$ up to $0.8 \mathrm{~g}$. The comparison shows the same results for lower numbers of stories while the increment is higher for the cases with eight stories (Fig. 9, right). In all cases, the values are divided by the cost of the building A at $S_{e}(g)=0$. The maximum spectral amplitudes considered in the various cases were relative to the maximum acceptable size of columns and beams.
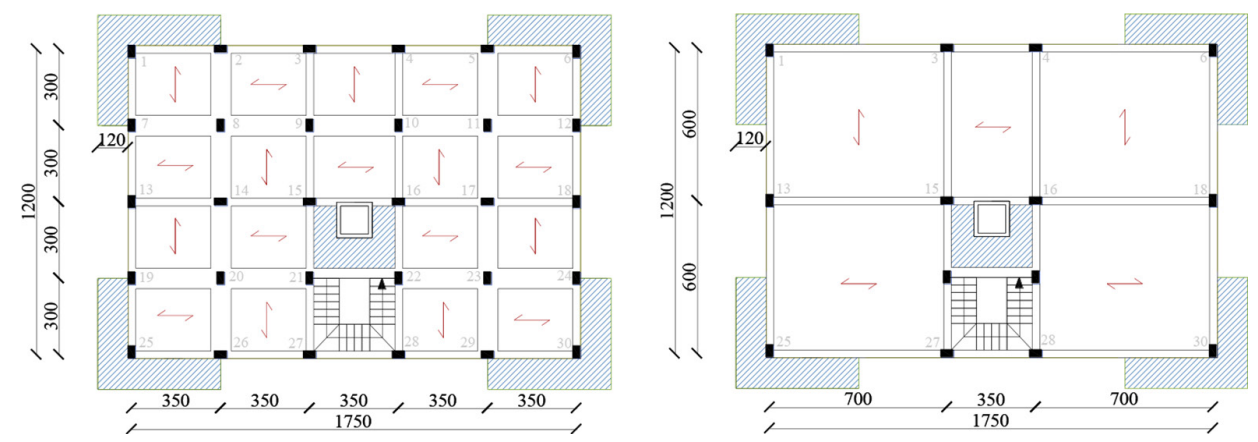

Figure 8: Plans of building A (left) and building B (right).
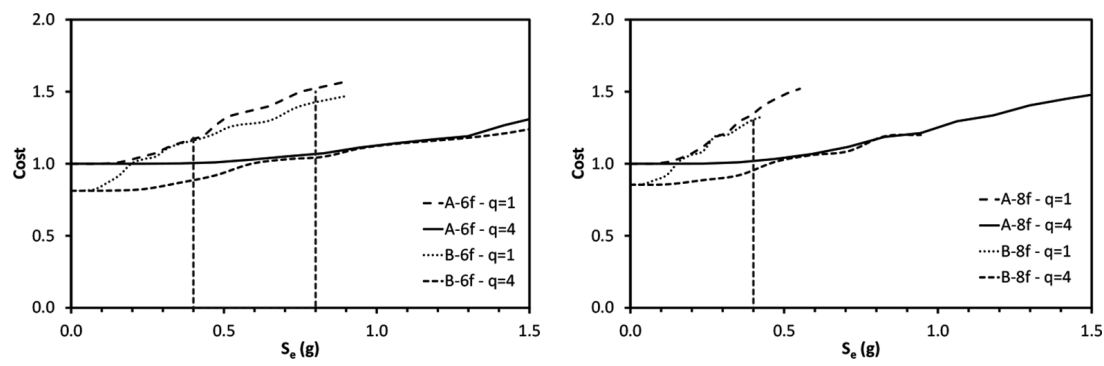

Figure 9: Construction cost for the six storied (left) and eight storied (right) buildings A and B with $q=1$ and $q=4$. 
A maximum design spectral value $S_{d, \max }$ could be suggested, based on economic and architectural considerations. This is a function of the materials and structural type and can be individualized as the value of $S_{d}$ for which the cost increases significantly. For example, in Fig. 9, the two values $0.4 \mathrm{~g}$ and $0.8 \mathrm{~g}$ were individualized for the case study. If the elastic spectral amplitude $S_{e}(T)$ at the fundamental period $T$ of the structure is lower than $S_{d, \max }$, then the elastic value should be assumed as the design value (Fig. 10). If not, $S_{d, \max }$ should be assumed as the design spectral value, the needed behavior factor could be considered, and the structure should be able to dissipate energy accordingly. This behavior factor should depend on the elastic spectral amplitude, i.e. on the seismic hazard at the site and on the fundamental period of vibration of the structure. Hence, it is variable also for a given response spectrum. This procedure is usual when designing base isolated buildings, for which the period is chosen in order to reach a spectral amplitude low enough to design the superstructure in the elastic range [11].

For example, assuming $S_{d, \max }=0.4 \mathrm{~g}$ as maximum elastic design value, it would be possible to design with $q=1$ in about $15 \%$ of the Italian territory for $T_{N C R}=2475$ years and in about $50 \%$ for $T_{N C R}=475$. With $S_{d, \max }=0.8 \mathrm{~g}$ the previous percentage rises up to $50 \%$ and $90 \%$, respectively (Fig. 11) [12]. Obviously, $S_{d, \max }$ should be chosen as a function of the

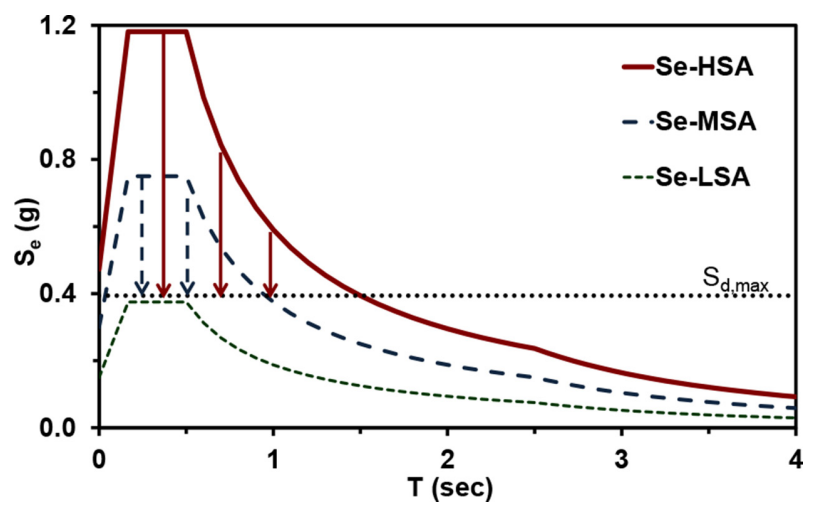

Figure 10: Ductility demand variable with the spectral amplitude.

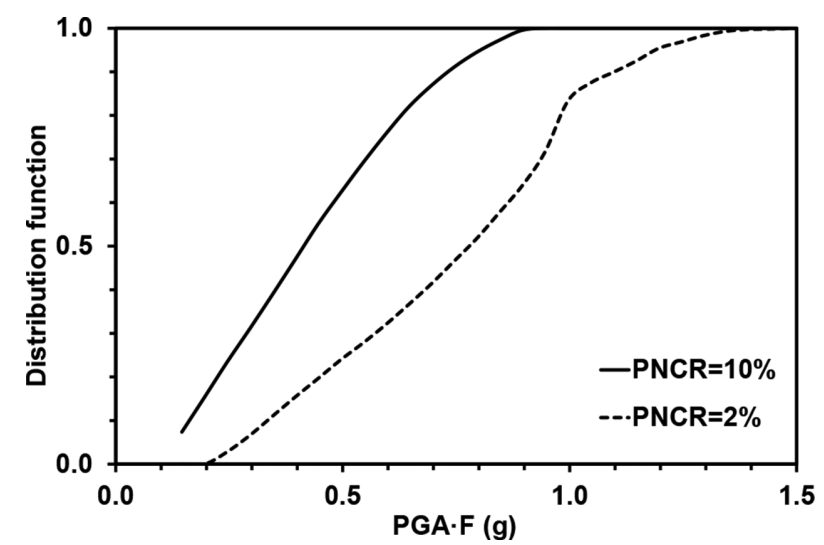

Figure 11: Distribution functions of maximum elastic spectral amplitude on rigid soil in the Italian territory for $P_{N C R}=10 \%$ and $P_{N C R}=2 \%$. 

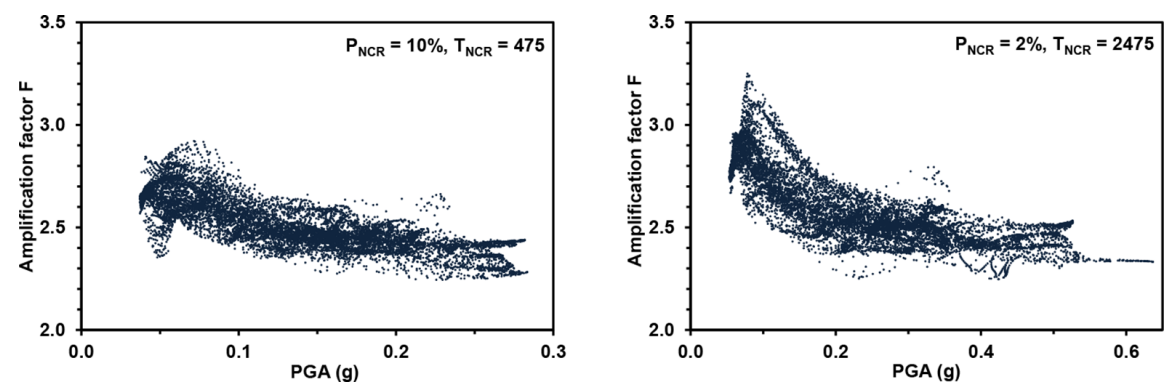

Figure 12: Couples $F-P G A$ in the Italian territory for $P_{N C R}=10 \%$ and $2 \%$.

structural type. It is important pointing out that also when designing with $q=1$ the structural conception and the details should guarantee a ductile behavior of the structure and all its components when their elastic limit is exceeded. Therefore, the structure and its components should have a suitable dissipation capacity. A level of ductility sufficient to support without collapse the design seismic actions amplified by 1.5 could be proposed.

Structural details usually prescribed for buildings in seismic areas are required in the so-called very low seismicity areas. For the identification of such areas, seismic codes refer to the value of the maximum acceleration on rigid soil relative to $T_{N C R}=475$ years. For example, $P G A=0.05 \mathrm{~g}$ is that assumed in the Italian technical code.

A more recent definition considers also the soil amplification factor $S$, and the limit value could become $P G A \cdot S=0.075 \mathrm{~g}$ (for $T_{R}=475$ years). The parameter $P G A \cdot S$ accounts for the soil amplification. As a result, the seismicity level will depend also on the foundation laying. Hence, two buildings very close to each other, with different foundation laying, could be in different seismic areas.

Both $P G A$ and $P G A \cdot S$ are relative to the ground motion at the bedrock and on the surface, respectively, but do not account for the effects on the structures. To do that, the parameter $P G A \cdot F \cdot S$ is certainly more representative. The parameter $F$, which is assumed constant and equal to 2.5 in the Eurocode 8 , is actually a function of the site and $P G A$; it increases when $P G A$ decreases. In Fig. 12 the couples $F-P G A$ are plotted for the Italian territory. Starting from the previous limit value of $P G A \cdot S$, the very low seismicity areas could be defined by the condition $P G A \cdot F \cdot S \leq 0.20 \mathrm{~g}$ (for $T_{N C R}=475$ years). Obviously, all these limit values should be changed if we refer to $T_{N C R}=2475$ years.

\section{SEISMIC INPUT IN THE RETROFIT OF EXISTING BUILDINGS}

The values of the seismic actions provided by the codes, and obtained by means of PSHA or DSHA, can be certainly used in the design of new buildings. For existing buildings, instead, achieving a similar level of protection is often impossible. Actually, most of the existing buildings have been designed with reference to seismic standards less severe than the current ones or even without taking into account the seismic actions. Hence, their complete seismic retrofit is very difficult, or even impossible, for technological and economic reasons, and a level of protection lower than that required for new constructions is usually accepted for them. As a result, an important difference in the base hazard evaluation, for example, between the values provided by the probabilistic and deterministic approaches, would be insignificant in practice.

For the historical structures, it is better to choose first the retrofit intervention with regard to the original structural design and the architectural and historical values, and then accept it if 
the degree of improvement achieved is higher than a minimum acceptable one, which should be fixed by the codes and depends on the risk we can accept. It is worth pointing out that for the rehabilitation of historical structures, designed without accounting for the seismic actions, and hence very vulnerable even to moderate events, the traditional techniques, based on the increasing of strength and ductility, are not suitable. Base isolation could be very useful, because it aims at the reduction of seismic actions, thus avoiding significant damages to the structure and its contents even under strong earthquakes, and presents very low interference with the structure itself. Innovative solutions for the application of seismic isolation to cultural heritage structures have already been proposed, which allow the realization of an isolation system under the foundations of the building, without touching the building itself [13,14].

The absence of apparent damage or the presence of low damage in buildings after seismic events do not guarantee against future earthquakes and also those buildings should be retrofitted. Often the structure is useful from an architectural point of view but able to support only very low seismic actions. In these cases, seismic isolation could be the best solution and the highest level of protection should be pursued by referring to the higher seismic action, i.e. to a lower value of $P_{\mathrm{NCR}}$.

\section{CONCLUSIONS}

Our knowledge about earthquakes and their effects on the structures is still quite low and the safety level of our structures is not satisfactory. For a suitable seismic prevention policy, the maximum earthquake intensity at each site should be assumed in the structural design, deduced from the probabilistic and/or deterministic approach, the first one usually giving higher values of the seismic acceleration.

Structures should be able to support the actions in the elastic range, in order to be operative also during and just after the strongest earthquakes at the site and to avoid heavy reconstruction costs. This is certainly possible in medium- and low-seismicity areas for all the structures and determine an acceptable increase of the construction cost. In more detail, an economic analysis, carried out on a reinforced concrete building, showed low increment of the structural construction cost with an elastic design up to $S_{e}=0.4 \mathrm{~g}$ and certainly acceptable up to $S_{e}=0.8 \mathrm{~g}$ for buildings up to six stories. This is not true for taller buildings but in these cases, different structural types should be used.

Strategic and important structures should be able to support the maximum seismic event without damage also in HSA. The inelastic behavior and the capacity to dissipate energy could be taken into account for normal buildings in HSA, for which a certain level of damage could be accepted without collapse. In this case, a lower elastic capacity could be accepted but a particular attention should be paid to the inelastic behavior and the dissipation capacity of the structure and hence to the structural details.

\section{REFERENCES}

[1] Bazzurro, P., Alexander, D., Clemente, P., Comerio, M., De Sortis, A., Filippou, F., Goretti, A., Jorjani, M., Mollaioli, F., Mosalam, K., Price, H.J., Court, C.P., Schotanus, M. \& Stewart, J., Learning from Earthquakes. The Mw 6.3 Abruzzo, Italy, Earthquake of April 6, 2009, EERI Special Earthquake. Report, 2009.

[2] Çelebi, M., Bazzurro, P., Chiaraluce, L., Clemente, P., Decanini, L., De Sortis, A., Ellsworth, W., Gorini, A., Kalkan, E., Marcucci, S., Milana, G., Mollaioli, F., Olivieri, M., Paolucci, R., Rinaldis, D., Rovelli, A., Sabetta, F. \& Stephens, C., Recorded motions of the Mw6.3 April 6, 2009 L'Aquila (Italy) earthquake and implications for building structural damage: a review. Earthquake Spectra, EERI, 26(3), pp. 651-684, 2010. doi: http://dx.doi.org/10.1193/1.3450317 
[3] Pozzati, P., Proliferation of regulations and technicality. AICAP News in Industria italiana del cemento, 3, pp. 3-5, 1993.

[4] Iervolino, I., Probabilità e salti mortali: le insidie della validazione dell'analisi di pericolosità attraverso l'occorrenza di singoli terremoti. Progettazionesismica, 2, pp. 37-43, IUSS Press, 2012.

[5] Abrahamson, N.A., Seismic hazard assessment: problems with current practice and future developments. Proc. of First European Conf. on Earth. Eng. and Seismology, Geneva, Switzerland, 2006. http://www.ce.memphis.edu/7137/PDFs/Abrahamson/ Abrahamson_2006.pdf

[6] Panza, G., Romanelli, F. \& Vaccari, F., Seismic wave propagation in laterally heterogeneous anelastic media: theory and applications to seismic zonation. Advances in Geophysics, 43, pp. 1-95, 2001. doi: http://dx.doi.org/10.1016/s0065-2687(01)80002-9

[7] Zuccolo, E., Vaccari, F., Peresan, A. \& Panza, G., Neo-deterministic and probabilistic seismic hazard assessment: a comparison over the Italian territory. Pure Appl. Geophys., 168, pp. 69-83, 2011. doi: http://dx.doi.org/10.1007/s00024-010-0151-8

[8] Rinaldis, D. \& Clemente, P., Seismic input characterization for some sites in Italy. Earthquake Resistant Engineering Structures IX, eds. C.A. Brebbia \& S. Hernández, WIT Transactions on Eng. Sciences, Vol. 79, pp. 13-21, 2013. doi: http://dx.doi. org/10.2495/eres 130021

[9] Saitta, F., Bongiovanni, G., Buffarini, G., Clemente, P, Martelli, A., Marzo, A., Marghella, G., Indirli, M. \& Poggianti, A., Behaviour of industrial buildings in the Pianura Padana Emiliana earthquake. In Focus on The Pianura Padana Emiliane Earthquake Energia, Ambiente e Innovazione, No. 4-5, parte II, pp. 47-57, ENEA: Roma, 2012.

[10] Clemente, P., Rinaldis, D., Bongiovanni, G. \& Saitta, F., What information is needed to design anti-seismic structures? Earthquake Resistant Engineering Structures IX, eds. C.A. Brebbia \& S. Hernández, WIT Transactions on Eng. Sciences, Vol. 79, pp. 3-11, 2013. doi: http://dx.doi.org/10.2495/eres130011

[11] Clemente, P. \& Buffarini, G., Base isolation: design and optimization criteria. Seismic Isolation and Protective Systems. Mathematical Science Publisher, 1(1), pp. 17-40, 2010. doi: http://dx.doi.org/10.2140/siaps.2010.1.17

[12] Bongiovanni, G., Buffarini, G., Clemente, P. \& Saitta, F., Building in seismic areas: towards a new prevention policy. 15th World Conf. on Earth. Eng., 15WCEE (September 24-28, Lisbon), Paper No. 0672, 2012.

[13] Clemente, P. \& De Stefano, A., Application of seismic isolation in the retrofit of historical buildings. Earthquake Resistant Engineering Structures, eds. C.A. Brebbia \& M. Maugeri, WIT Transactions on the Built Environment, Vol. 120, pp. 41-52, 2011. doi: http://dx.doi.org/10.2495/eres110041

[14] Clemente, P., De Stefano, A. \& Renna, S., Isolation system for existing buildings. Proc. of the 12th World Conf. On Seismic Isolation, Energy Dissipation and Active Control of Structures - 12WCSI (September 20-23, Sochi, Russia), ASSISi, 2011. 